\title{
Personalized medicine: the enabling role of nanotechnology
}

\author{
"Nanotechnology-enabled theranostic approaches may indeed \\ become a key driver in harmonizing the needs of the various \\ stakeholders by allowing cost-effective delivery and monitoring \\ of drug efficiency and safety, and close-meshed high-quality data \\ collection."
}

First draft submitted: 14 August 2015; Article accepted for publication: 27 August 2015;
Published online: 11 December 2015

Keywords: big data $\bullet$ data-driven $\bullet$ diagnostics $\bullet$ monitoring $\bullet$ nanomedicine $\bullet$ patientcentered care $\bullet$ personalized health $\bullet$ precision medicine

Personalized medicine has recently taken center stage in cancer research and has led to a number of therapeutic success stories in oncological practice. Cancer is an immensely complex, heterogeneous disease that is caused by the interplay of multiple intrinsic (genetic predisposition) and extrinsic (lifestyle) factors. As cancer progresses, genetic drifts in the cell population typically result in heterogeneity with regard to cell antigenicity, differentiation state, proliferation rate, invasiveness, metastatic potential and response to chemotherapeutics. It has been increasingly recognized that cancer is a heterogeneous, evolving and adaptive malady that requires personalization of the treatment, as to the extent where the right drug is administered to the right patient with the right dose at the right time. Given the system complexity and the vastly limited understanding of the underlying pathophysiology, however, this poses a constitutive challenge to modern medicine (multiparameter optimization problem with many unknowns).

In recent years, analysis of somatic mutations in cancer genomes has led to the identification of a compendium of aberrations that confer cancer development and has in turn enabled development of drugs that specifically target hallmarks of neoplasia [1]. A range of small-molecule inhibitors and therapeutic antibodies have been developed to target overexpressed proteins, driving the exceedingly rapid translation of genomic discoveries into clinical end points. Selection of patient subsets (so-called responders) out of bigger patient cohorts based on genomic data has proven to be highly beneficial with regard to drug efficacy and patient outcome and has ushered in a new era, the era of precision medicine. Although perceived with much excitement by the scientific community, personalized therapy heavily relies on initial genomic profiling and the constitutive monitoring of biomarkers which require costefficient analysis of massive amounts of data, and hence few personalized medicines have entered clinical routine [2].

While a few years back the challenges in this field were scientific in nature, with the maturation of the '-omics' sciences, it now seems that the major barriers are increasingly related to effective clinical implementation and socioeconomics [3,4]. Personalized medicine requires a re-thinking and transition away from the 'one drug to fit all' idea. Every day, millions of people take medications that will not only not help but in the worst case even harm them, hence causing a significant burden to the healthcare system. Still, these very same medications are the drugs that reach blockbuster status and bring the revenue that is so vital for a filled drug development pipeline and thus are criti-
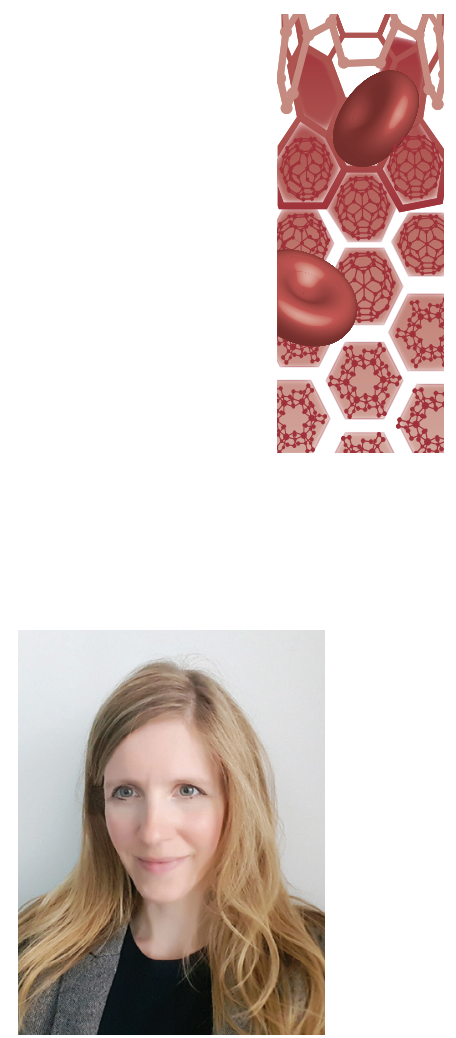

Inge K Herrmann Author for correspondence: Department Materials Meet Life, Swiss Federal Laboratories for Materials Science \& Technology (Empa), Lerchenfeldstrasse 5, 9014 St. Gallen, Switzerland Tel.: +41 (0)587657153 inge.herrmann@empa.ch

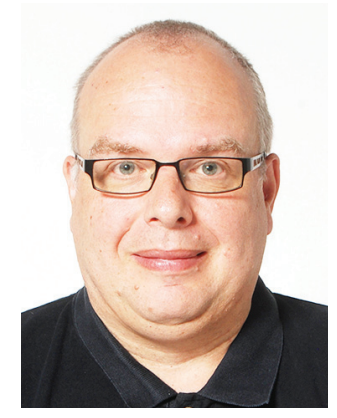

Matthias Rösslein Department Materials Meet Life, Swiss Federal Laboratories for Materials Science \& Technology (Empa), Lerchenfeldstrasse 5, 9014 St. Gallen, Switzerland
Future Medicine part of 
cal for a healthy pharmaceutical industry. For pharmaceutical industry, personalized medicine in its current form essentially becomes equivalent to a shrunken market and diminished revenue potential. Clearly, the incentives for the stakeholders are severely misaligned, similar to what has been observed previously for the development of new antibiotics: While the slow adoption of new antibiotics may be appropriate for public health maximization to delay development of resistance, it is the key factor that drives the poor economics for companies. Moreover, tailored drugs are only useful if tailored diagnostics can identify appropriate patients, hence posing dual burdens of large, expensive clinical trials and diminished revenue potential on pharmaceutical industry [3]. Classical clinical trials may not be appropriate anymore. Schork recently discussed the concept of one-person trials, a different type of clinical trial that focuses on individual rather than averaged responses to therapy [5]. While this is being done for rare diseases out of necessity, the amount of data that is collected is not usually sufficient to make hypotheses about the drug's mechanism that could later on form the basis for a generalized understanding. As pointed out by Schork, one-patient trials hold a distinct advantage over classical trials, namely, that the patients involvement will not only help future generations but the treatment will be vetted for the actual study patients. If done properly, such one-patient trials could directly help the involved patient as well as form the ground work (i.e., high-quality data) for the treatment of future generations. However, implementation of one-patient trials also heavily depends on the physicians' incentives. To date, physicians get disproportionally higher rates for procedure-oriented services rather than for evaluation and monitoring of the outcome [3]. This leads to a financial disincentive to run diagnostic tests to prescreen patients only to find that further treatment is ineffective or unnecessary. At the same time, this has led to the resumption of the ethically highly controversial debate whether patients should pay for their participation in clinical research [6].

\section{"Personalized medicine is an interdisciplinary challenge where theranostic approaches and sophisticated data management are likely to take center stage to enable personalized treatment at acceptable cost-benefit ratios."}

Between all these conflicting priorities, there are a number of scientific opportunities that may contribute to the easing of the situation by opening new markets, leading to a redistribution of costs and revenues between the various stakeholders. Technological solutions for the accelerated discovery of biomarkers, the engineering of improved drug carriers, the development of point-of-care diagnostic tests and monitoring devices, and appropriate data management approaches may turn precision medicine into profitable endeavors for all parties involved [7]. Particularly, nanotechnology-enabled approaches have demonstrated astonishing potential to transform medicine and may enable smoother transitions from the 'one drug to fit all' state to highly personalized treatment modalities. The advent of nanotechnology has brought with it a multitude of different diagnostic, therapeutic and theranostic approaches [8,9]. Nanosized carriers can be engineered bottom-up to a highly customizable extent, hence enabling much needed cost-effective theranostic approaches by integrating imaging (monitoring) and therapy, and allowing targeted delivery of drugs by superimposing natural biodistribution.

Safe-by-design delivery of drugs by means of nanosized carriers may significantly impact drug development and treatment by reducing the pressure for all stakeholders. Currently, the US FDA records a 95\% failure rate for drug approval due to safety and efficacy issues; hence, there is an urgent need to reduce the risk for pharmaceutical industry and patients and make both drug development and use safer. Specifically, nanosized reporter probes may be employed to deliver and monitor active ingredients and provide immediate (online) feedback on the therapeutic effectiveness of the treatment [10-15]. Additionally, the development of generic nanosized trigger-responsive carriers holds great potential to site specifically deliver active ingredients in a responsive manner (that is only under certain conditions), thus reducing toxicity and off-target effects [16,17]. The use of nanosized drug carriers opens the opportunity to deliver drugs in a way that is tailored not only to the nanoscale (gene and protein expression level) but also to the mesoscale (microenvironment), as recently reviewed by Nuria and colleagues [18].

Nanotechnology-enabled carrier and reporter systems may assist in the collection of patient-specific data noninvasively by giving access to a previously inaccessible spatiotemporal resolution (mesoscale imaging of the microenvironment). Additionally, nanotechnology-enabled point-of-care systems may assist in close-meshed data collection as well as compliance monitoring at reduced cost, hence reducing the overall burden to the healthcare system. Point-of-need compatible, easy-to-use (paper-based) diagnostic devices and naked-eye detection of biomarkers are likely to transform data collection $[9,19,20]$. Constitutive noninvasive monitoring is particularly relevant for a range of conditions, including inflammatory and autoimmune diseases, and may in turn have direct impact on the 
patient's quality of life at acceptable cost-benefit ratios. Here, physicians and patients will assume a more active role in data collection and monitoring of treatment success, most likely resulting in increased patient engagement and compliance [21].

Personalized medicine is an interdisciplinary challenge where theranostic approaches and sophisticated data management are likely to take center stage to enable personalized treatment at acceptable cost-benefit ratios. Despite the high societal value, the transition from classical clinical trials to one-patient trials and personalized treatment still faces significant obstacles, most of them due to misaligned incentives [4]. Personalized treatment could become a profitable market, if we work out where that profit will come from as a society (and not as individual stakeholders) and if we

\section{References}

1 Stegh AH. Toward personalized cancer nanomedicine - past, present, and future. Integr. Biol. 5(1), 48-65 (2013).

2 Chin L, Andersen JN, Futreal PA. Cancer genomics: from discovery science to personalized medicine. Nat. Med. 17(3), 297-303 (2011).

3 Davis JC, Furstenthal L, Desai AA et al. The microeconomics of personalized medicine: today's challenge and tomorrow's promise. Nat. Rev. Drug Discov. 8(4), 279-286 (2009).

4 Hood L, Friend SH. Predictive, personalized, preventive, participatory (P4) cancer medicine. Nat. Rev. Clin. Oncol. 8(3), 184-187 (2011).

5 Schork NJ. Time for one-person trials. Nature 520(7549), 609-611 (2015).

6 Emanuel EJ, Joffe S, Grady C, Wendler D, Persad G. Clinical research: should patients pay to play? Sci. Transl. Med. 7(298), 298ps16 (2015).

7 Hamburg MA, Collins FS. The path to personalized medicine. N. Engl. J. Med. 363(4), 301-304 (2010).

8 Jain KK. Role of nanobiotechnology in developing personalized medicine for cancer. Technol. Cancer Res. Treat. 4(6), 645-650 (2005).

9 Howes PD, Chandrawati R, Stevens MM. Bionanotechnology. Colloidal nanoparticles as advanced biological sensors. Science 346(6205), 1247390 (2014).

10 Theek B, Rizzo LY, Ehling J, Kiessling F, Lammers T. The theranostic path to personalized nanomedicine. Clin. Transl. Imaging 2(1), 66-76 (2014).

11 Schroeder A, Heller DA, Winslow MM et al. Treating metastatic cancer with nanotechnology. Nat. Rev. Cancer 12(1), 39-50 (2012). put incentives in place. Nanotechnology-enabled theranostic approaches may indeed become a key driver in harmonizing the needs of the various stakeholders by allowing cost-effective delivery and monitoring of drug efficiency and safety, and close-meshed high-quality data collection.

\section{Financial \& competing interests disclosure}

The authors have no relevant affiliations or financial involvement with any organization or entity with a financial interest in or financial conflict with the subject matter or materials discussed in the manuscript. This includes employment, consultancies, honoraria, stock ownership or options, expert testimony, grants or patents received or pending, or royalties.

No writing assistance was utilized in the production of this manuscript.

12 Ojha T, Rizzo L, Storm G, Kiessling F, Lammers T. Imageguided drug delivery: preclinical applications and clinical translation. Expert Opin. Drug Deliv. 12(8), 1203-1207 (2015).

13 Xie J, Lee S, Chen XY. Nanoparticle-based theranostic agents. Adv. Drug Deliv. Rev. 62(11), 1064-1079 (2010).

14 Cai WB, Chen XY. Nanoplatforms for targeted molecular imaging in living subjects. Small 3(11), 1840-1854 (2007).

15 Wu X, Sun X, Guo Z et al. In vivo and in situ tracking cancer chemotherapy by highly photostable NIR fluorescent theranostic prodrug. J. Am. Chem. Soc. 136(9), 3579-3588 (2014).

16 Mura S, Nicolas J, Couvreur P. Stimuli-responsive nanocarriers for drug delivery. Nat. Mater. 12(11), 991-1003 (2013).

17 Torchilin VP. Multifunctional, stimuli-sensitive nanoparticulate systems for drug delivery. Nat. Rev. Drug Discov. 13(11), 813-827 (2014).

18 Oliva N, Unterman S, Zhang Y, Conde J, Song HS, Artzi N. Personalizing biomaterials for precision nanomedicine considering the local tissue microenvironment. Adv. Healthc. Mater. 4(11), 1584-1599 (2015).

19 Martinez AW, Phillips ST, Whitesides GM, Carrilho E. Diagnostics for the developing world: microfluidic paperbased analytical devices. Anal. Chem. 82(1), 3-10 (2010).

20 Yetisen AK, Akram MS, Lowe CR. Paper-based microfluidic point-of-care diagnostic devices. Lab Chip 13(12), 2210-2251 (2013).

21 White CC, Fang D, Eung-Hun K, Lober WB, Yongmin K. Improving healthcare quality through distributed diagnosis and home healthcare (D/sub 2/H/sub 2/). Presented at: $1 s t$ Transdisciplinary Conference on Distributed Diagnosis and Home Healthcare, 2006. D2H2. Arlington, VA, USA, 2-4 April 2006. 